\title{
A Case of Schizophrenia with Chromosomal Microdeletion of 17p11.2 Containing a Myelin-Related Gene PMP22
}

\author{
Yuji Ozeki ${ }^{\mathrm{a}}$, Takeshi Mizuguchi ${ }^{\mathrm{b}}$, Naotsugu Hirabayashi ${ }^{\mathrm{c}}$, Masafumi Ogawa ${ }^{\mathrm{d}}$, Naomi Ohmura ${ }^{\mathrm{e}}$, \\ Miyuki Moriuchi $^{\mathrm{e}}$, Naoki Harada ${ }^{\mathrm{e}, \mathrm{f}}$, Naomichi Matsumoto ${ }^{\mathrm{b}, \mathrm{f}}$ and Hiroshi Kunugi ${ }^{*}$,a
}

\author{
${ }^{a}$ Department of Mental Disorder Research, National Institute of Neuroscience, National Center of Neurology and \\ Psychiatry, 4-1-1, Ogawahigashi, Kodaira, Tokyo, 187-8502, Japan \\ ${ }^{b}$ Department of Human Genetics, Yokohama City University Graduate School of Medicine, Yokohama, Japan \\ ${ }^{c}$ Department of Psychiatry, National Center Hospital for Mental, Nervous and Muscular Disorders, National Center of \\ Neurology and Psychiatry, Tokyo, Japan \\ ${ }^{d}$ Department of Neurology, National Center Hospital for Mental, Nervous, and Muscular Disorders, National Center of \\ Neurology and Psychiatry, Tokyo, Japan \\ ${ }^{e}$ Kyushu Medical Science Nagasaki Laboratory, Nagasaki, Japan \\ ${ }^{f}$ Solution-Oriented Research for Science and Technology, Japan Science and Technology Agency, Kawaguchi, Japan
}

\begin{abstract}
We report a patient with schizophrenia who had a chromosomal deletion of $17 \mathrm{p} 11.2$ containing a myelinrelated gene PMP22 by using comparative genomic hybridization (CGH) array and quantitative PCR. Since genetic linkage to $17 \mathrm{p} 11$, reduced expression of PMP22, and alterations in myelination have previously been reported, this report further suggests an etiological role of PMP22 in schizophrenia.
\end{abstract}

Keywords: Schizophrenia, PMP22, 17p11.2.

\section{INTRODUCTION}

Chromosomal aberrations found in patients with schizophrenia have provided possibly important insights into the molecular mechanism of the illness [1,2], although such aberrations are rare [3]. For example, 22q11 deletion syndrome (22q11DS) is a well known risk factor for schizophrenia [4]. DISC1, which is one of promising candidate genes of schizophrenia, was found as a disrupted gene by chromosomal translocation $[5,6]$. In this context, we have been screening chromosomal abnormalities by using the array comparative genomic hybridization (CGH) in 42 schizophrenia subjects and found a patient who had a chromosomal microdeletion of $17 \mathrm{p} 11.2$ containing the myelin-related gene, $P M P 22$. $P M P 22$ is causal to Charcot-Marie-Tooth neuropathy type 1 A (CMT1A) [7] and hereditary neuropathy with liability to pressure palsies (HNPP) [8]. Three copies of PMP22 (duplication) result in CMT1A, while one copy (deletion) in HNPP. Patients with CMT1A have reduced nerve conduction velocities [9]. HNPP is characterized by diverse sensory or motor nerve palsies which are often precipitated by minor trauma.

\section{CASE REPORT}

\section{Case History}

The proband was 32-year-old man who was the first child of reportedly unrelated parents. He had a surgical operation

\footnotetext{
*Address correspondence to this author at the Department of Mental Disorder Research, National Institute of Neuroscience, National Center of Neurology and Psychiatry, 4-1-1, Ogawahigashi, Kodaira, Tokyo, 187-8502, Japan; E-mail: hkunugi@ncnp.go.jp
}

for inguinal herniation when he was 2 months old. According to his mother, the development of his verbal communication was delayed. When he was 11 years old, he lost consciousness for a few minutes with no apparent external event inducing such loss of consciousness; however, no abnormality was detected with electroencephalography or the computed tomography (CT) scan of the head immediately after the loss of consciousness. The CT film is not available now because the brain CT scan was carried out more than 20 years ago in another hospital. He entered ordinary elementary and junior high schools without receiving any special education. Although he went on to enter a high school, he dropped out at the age of 17 years. When he was 19 years old, auditory hallucination and delusion of persecution developed. Six months later, he started antipsychotic treatment at a psychiatric clinic. Then he was introduced to our hospital to control his psychotic symptoms and adverse effects (tremor) induced by the antipsychotics with a clinical diagnosis of schizophrenia and mental retardation. Finally, his psychiatric symptoms and tremor were controlled by $100 \mathrm{mg}$ floropipamide, $2 \mathrm{mg}$ trihexyphenidyl, and $1 \mathrm{mg}$ biperiden. He had never showed motor paralysis or sensory disturbance. His intelligence quotient (IQ) was 42 by the Wechsler adult intelligence scale-revised (WAIS-R) [10] at the age of 29 years. Laboratory tests for blood and urine did not show any abnormality. His final diagnoses were schizophrenia and mental retardation, according to the structured interview of DSM-IV $[11,12]$. 
This study was approved by the ethics committee of the National Center of Neurology and Psychiatry, Tokyo, Japan. Written informed consent was obtained from the proband and his parents to perform chromosomal examination, and publish this case report.

\section{Family History}

His father was a professor of a university and his mother was a housewife with normal social function. Structural interview by Mini-International Neuropsychiatric Interview $[13,14]$ did not indicate any present or past psychiatric diagnosis in his parents. The proband had a younger sister who had no current or past history of psychiatric illness according to the parents.

\section{Array CGH Findings}

The proband participated in our ongoing screening of chromosomal abnormalities in a series of patients with schizophrenia. Chromosomal abnormalities were assessed with the array CGH method developed by Miyake et al. [15] using a newly developed 4.2K microarray with 4,235 FISHed BAC clones. A typical $1.4-\mathrm{Mb}$ microdeletion at 17 11.2, containing PMP22, was identified. The deletion was also confirmed by fluorescence in situ hybridization (FISH) analysis (Fig. 1).

\section{Quantitative PCR for Copy Number Variation}

We examined copy number of $P M P 22$ for the proband and his parents by the quantitative PCR of genomic DNA. TaqMan probes were chosen, and delta-delta $\mathrm{Ct}$ method was applied, according to a previous study [16]. Quantitative PCR was done with the ABI prism 7900 (Applied Biosystems, Foster city, CA, USA). A Delta-delta $\mathrm{Ct}$ value around 0.5 indicates that the copy number of the gene is one and the value around 1 means two copies. The delta-delta $\mathrm{Ct}$ values of the proband, his father, and his mother were $0.65,0.50$, and 1.06 , respectively, suggesting that the microdeletion of the proband was transmitted from his father.

\section{Neurological Assessment}

A clinical neurologist assessed neurological symptoms of the proband. However, no symptom of HNPP was apparent including abnormality of deep tendon reflex. A nerve conduction study was carried out for the proband; however, the result was within normal limit except for the distal motor latency of right tibial nerve. He does not show pes cavus or hammer toes. These findings are incongruent with the phenotype of PMP22 deletion described by Mouton et al. [17]. His father did not report any neurological symptoms, either, although a nerve conduction study was not carried out for him.

\section{DISCUSSION}

We report a case of schizophrenia who had a microdeletion of chromosome $17 \mathrm{p} 11.2$ containing a myelin-related gene, PMP22. To our knowledge, this is the first report of such a case. Chromosomal microdeletion was confirmed by FISH and RT-PCR in addition to the initial array CGH method. Thus it is unlikely that the detected deletion was an artifact, although the case we report here was asymptomatic with respect to HNPP and had no clear family history of HNPP. Mouton et al. [17] investigated 99 individuals with the $17 \mathrm{p} 11.2$ deletion in 22 families and found that fourteen individuals showed no symptom. Thus asymptomatic individuals like our case seem to be not rare.

It is possible that coexistence of schizophrenia and the deletion of $17 \mathrm{p} 11.2$ in the proband may have occurred by simple coincidence. The absence of psychiatric history in the proband's father, who had the same deletion, further supports such a possibility. However, it is also possible that incomplete penetrance of the genetic effect of the deletion may explain the discrepancy in psychiatric condition between the proband and his father. Indeed, several lines of evidence in addition to our case have suggested that PMP22 may play a role in the pathogenesis of schizophrenia. Dracheva et al. [18] reported that mRNA of PMP22 was reduced in the hippocampus and anterior cingulate cortex in post mortem brains of schizophrenia. PMP22 is highly expressed and plays a critical role in functions of oligodendrocytes, which accords with previous studies indicating the oligodendrocyte dysfunction in schizophrenia [19,20,21]. Decreased number of perineuronal oligodendrocytes was reported in the prefrontal cortex of patients with schizophrenia [22]. Transgenic mice which have oligodendrocyte dysfunction have increased levels of dopamine receptors and transporters [23]. Then defects in white matter can cause hyper-dopaminergic symptoms (e.g. delusion and hallucination). Reduced fraction anisotropy in white matter of patients with schizophrenia by a diffusion tensor imaging study [24] may due to oligodendrocyte dysfunction. Oligodendrocytes produce trophic factors such as brain derived neurotrophic factor (BDNF) [25] and neuregulins (NRGs) [26]. BDNF and NRG1 are believed in playing an important role in the etiology of schizophrenia. PMP22 dysfunction may decrease the function of BDNF [27] and NRG1 [28]. Finally, some genome wide linkage studies provide evidence for linkage to 17p11.2-q25.1 in schizophrenic pedigrees [29,30,31]. To further elucidate the possible role of PMP22 in schizophrenia, molecular genetic studies and psychiatric examination on individuals with CMT1A and HNPP are warranted.

\section{CONCLUSIONS}

In conclusion, we found a patient with schizophrenia who had a chromosomal microdeletion of $17 \mathrm{p} 11.2$ containing $P M P 22$, a gene critical to oligodendrocyte functions. Since reduced expression of $P M P 22$, alterations in oligodendrocyte functions, and genetic linkage to $17 \mathrm{p} 11$ have previously been reported, our case further supports an etiological role of PMP22 in schizophrenia.

\section{ACKNOWLEDGEMENTS}

This study was supported by Health and Labor Sciences Research Grants (Research on Psychiatric and Neurological Diseases and Mental Health) from the Ministry of Health and Welfare, Grant-in-Aid for Scientific Research from the Japan Society for the Promotion of Science (JSPS), and Grant-in-Aid for Scientific Research on Priority Areas. 


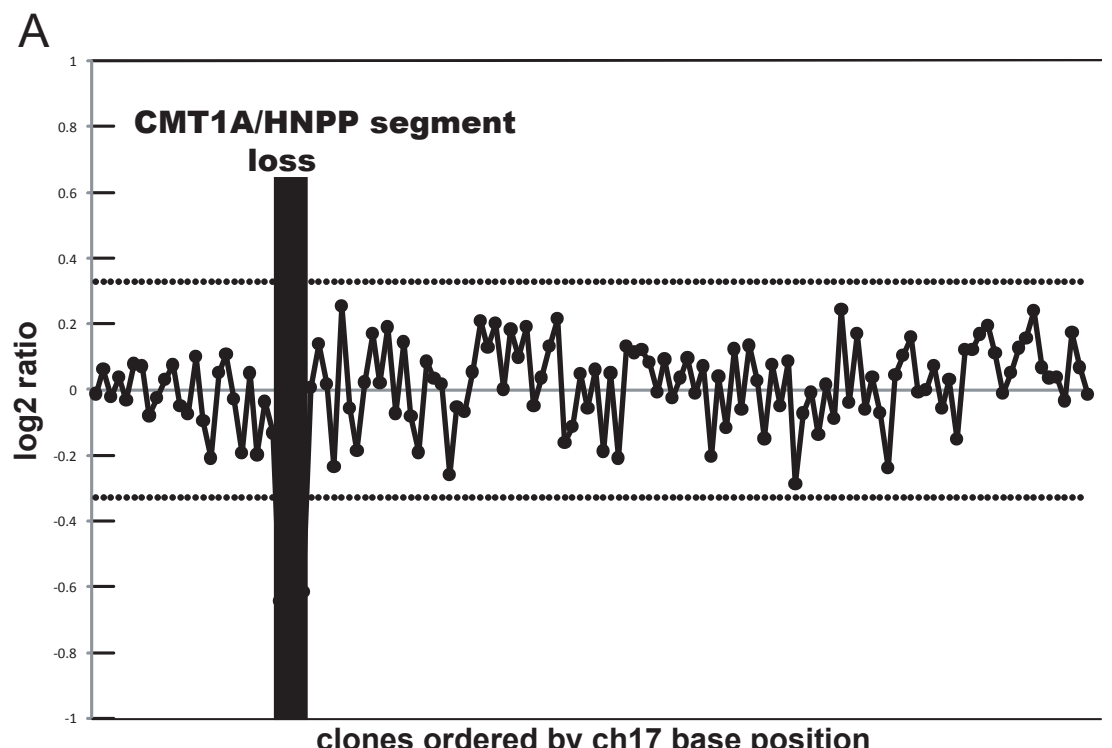

B

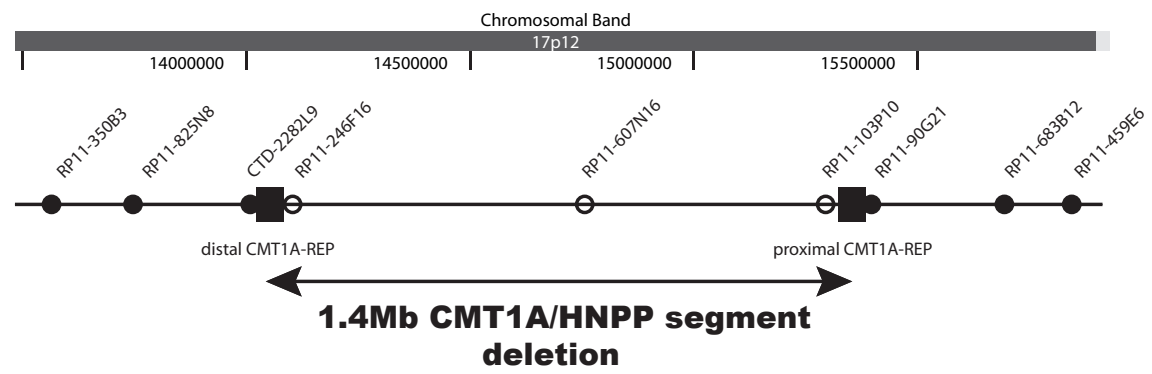

C
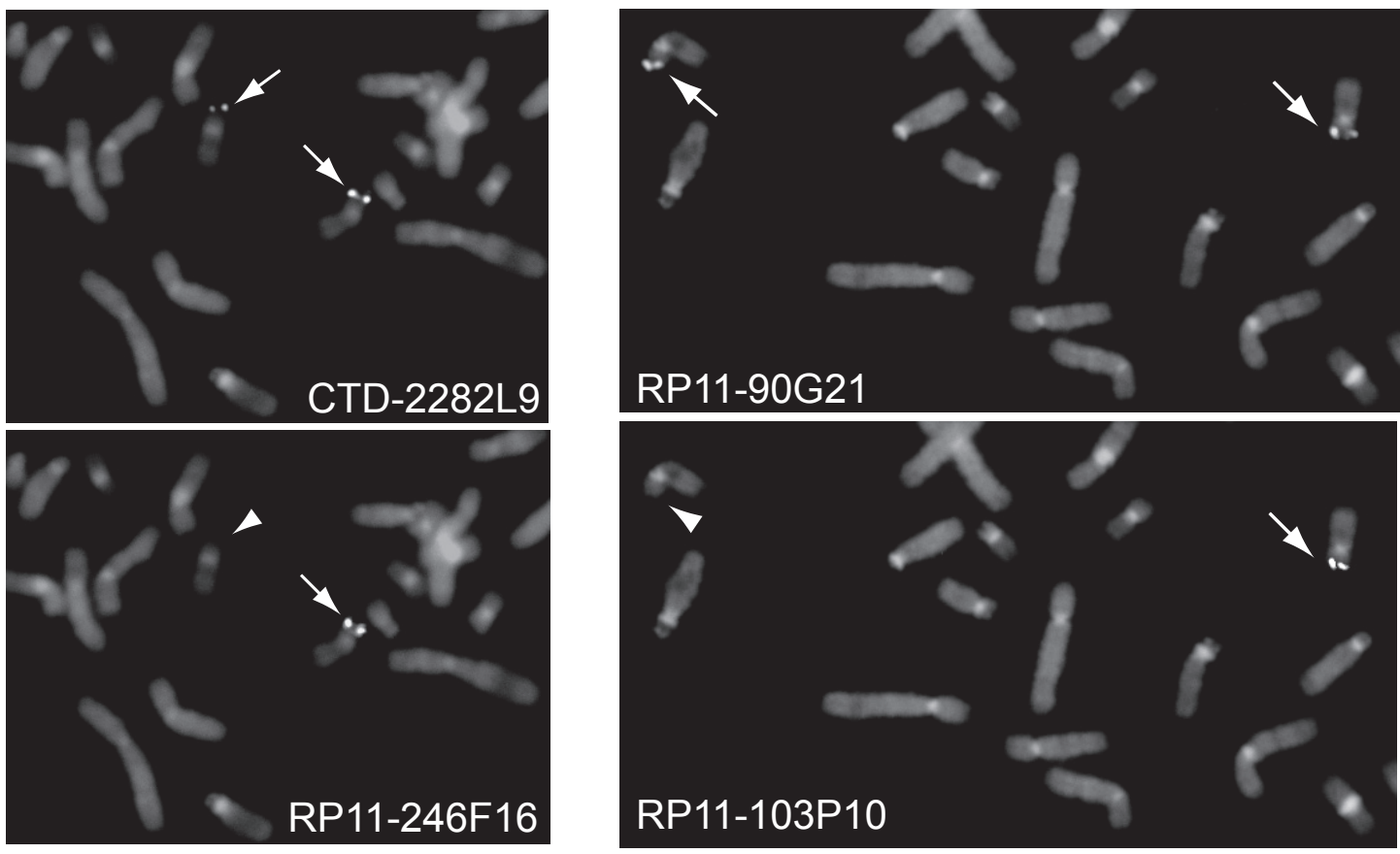

Fig. (1). (A) Microarray CGH analysis showing deletions at CMT1A/HNPP locus. (B) Schematic presentation of BAC clones delineating the CMT1A/HNPP deletion in the patient. Open circle: BAC clone deleted in the patient, closed circle: BAC clone not deleted in the patient. Closed square: low copy repeat which may have mediated genomic rearrangements. A common 1.4-Mb deletion of HNPP occurs between proximal CMT1A-REP and distal CMT1A-REP. (C,D) BAC FISH analysis in the proband. Arrows show intact signals in 17p11.2 region. Arrow heads show the loss of signal in 17p11.2 region. Clone positions are indicated in Fig. (1B). 


\section{REFERENCES}

[1] MacIntyre DJ, Blackwood DH, Porteous DJ, Pickard BS, Muri WJ. Chromosomal abnormalities and mental illness. Mol Psychiatry 2003; 8: 275-87.

[2] Bassett AS, Chow EW, Weksberg R. Chromosomal abnormalities and schizophrenia. Am J Med Genet 2000; 97: 45-51.

[3] Kunugi H, Lee KB, Nanko S. Cytogenetic findings in 250 schizophrenics: evidence confirming an excess of the $\mathrm{X}$ chromosome aneuploidies and pericentric inversion of chromosome 9. Schizophr Res 1999; 40: 43-7.

[4] Millar JK, Wilson-Annan JC, Anderson S, et al. Disruption of two novel genes by a translocation co-segregating with schizophrenia. Hum Mol Genet 2000; 9: 1415-23.

[5] Murphy KC, Own MJ. The behavioural phenotype in velo-cardiofacial-syndrome. Am J Hum Genet 1997; 61: A5.

[6] Blackwood DH, Fordyce A, Walker MT, St Clair DM, Porteous DJ, Muir WJ. Schizophrenia and affective disorders--cosegregation with a translocation at chromosome 1q42 that directly disrupts brain-expressed genes: clinical and P300 findings in a family. Am J Hum Genet 2001; 69: 428-33.

[7] Lupski JR, de Oca-Luna RM, Slaugenhaupt S, et al. DNA duplication associated with Charcot-Marie-Tooth disease type 1A. Cell 1991; 66: 219-232.

[8] Chance PF, Alderson MK, Leppig KA, et al. DNA deletion associated with hereditary neuropathy with liability to pressure palsies. Cell 1993; 72: 143-51.

[9] Choi BO, Kim J, Lee KL, Yu JS, Hwang JH, Chung KW. Rapid diagnosis of CMT1A duplications and HNPP deletions by multiplex microsatellite PCR. Mol Cells 2007; 23: 39-48.

[10] Wechsler D, translated into Japanese by Shinagawa F, Kobayashi $\mathrm{S}$, Fujita K, Maegawa $\mathrm{H}$ Wechsler Adult Intelligence ScaleRevised Japanese version.; SACCESS BELL Co. Ltd., 1990

[11] American Psychiatric Association. translated into Japanese by Takahashi S, Ohno Y, Someya T. In Diagnostic and Statistical Manual of Mental Disorders 4th Ed. Japanese version.; Tokyo: Igaku-Shoin Ltd., 1996.

[12] First MB, Spitzer RL, Gibbon M, Williams JBW, translated into Japanese by Kitamura T, Okano T. Structured Clinical Interview for DSM-IV Axis I Disorders (SCID). Japanese version.; Tokyo: NIPPON HYORONSHA Co. Ltd., 2003.

[13] Otsubo T, Tanaka K, Koda R, et al. Reliability and validity of Japanese version of the Mini-International Neuropsychiatric Interview. Psychiatry Clin Neurosci 2005; 59: 517-526.

[14] Sheehan DV, Lecrubier Y, Sheehan KH, et al. The MiniInternational Neuropsychiatric Interview (M.I.N.I.): the development and validation of a structured diagnostic psychiatric interview for DSM-IV and ICD-10. J Clin Psychiatry 1998; 59(Suppl 20): 22-57.

[15] Miyake N, Shimokawa O, Harada N, et al. BAC array CGH reveals genomic aberrations in idiopathic mental retardation. Am J Med Genet A 2006; 140: 205-11.
[16] Aarskog NK, Vedeler CA. Real-time quantitative polymerase chain reaction. A new method that detects both the peripheral myelin protein 22 duplication in Charcot-Marie-Tooth type 1A disease and the peripheral myelin protein 22 deletion in hereditary neuropathy with liability to pressure palsies. Hum Genet 2000; 107: 494-8.

[17] Mouton P, Tardieu S, Gouider R, et al. Spectrum of clinical and electrophysiologic features in HNPP patients with the $17 \mathrm{p} 11.2 \mathrm{de}-$ letion. Neurology 1999; 52: 1440-46.

[18] Dracheva S, Davis KL, Chin B, Woo DA, Schmeidler JH, Aroutunian V. Myelin-associated mRNA and protein expression deficits in the anterior cingulate cortex and hippocampus in elderly schizophrenia patients. Neurobiol Dis 2006; 21: 531-40.

[19] Haroutunian V, Katsel P, Dracheva S, Stewart DG, Davis KL. Variations in oligodendrocyte-related gene expression across multiple cortical regions: implications for the pathophysiology of schizophrenia. Int J Neuropsychopharmacol 2007; 10: 565-73.

[20] Davis KL, Stewart DG, Friedman JI, et al. White matter changes in schizophrenia: evidence for myelin-related dysfunction. Arch Gen Psychiatry 2003; 60: 443-56.

[21] Hakak Y, Walker JR, Li C, et al. Genome-wide expression analysis reveals dysregulation of myelination-related genes in chronic schizophrenia. Proc Natl Acad Sci USA 2001; 98: 4746-51.

[22] Vostrikov VM, Uranova NA, Orlovskaya DD. Deficit of perineuronal oligodendrocytes in the prefrontal cortex in schizophrenia and mood disorders. Schizophr Res 2007; 94: 273-80.

[23] Roy K, Murtie JC, El-Khodor BF, et al. Loss of erbB signaling in oligodendrocytes alters myelin and dopaminergic function, a potential mechanism for neuropsychiatric disorders. Proc Natl Acad Sci USA 2007; 104: 8131-6.

[24] Shergill SS, Kanaan RA, Chitnis XA, et al. A diffusion tensor imaging study of fasciculi in schizophrenia. Am J Psychiatry 2007; 164: 467-73.

[25] Dougherty KD, Dreyfus CF, Black IB. Brain-derived neurotrophic factor in astrocytes, oligodendrocytes, and microglia/macrophages after spinal cord injury. Neurobiol Dis 2000; 7: 574-85.

[26] Deadwyler GD, Pouly S, Antel JP, Devries GH. Neuregulins and erbB receptor expression in adult human oligodendrocytes. Glia 2000; 32: 304-12.

[27] Hashimoto T, Lewis DA. BDNF Val66Met polymorphism and GAD67 mRNA expression in the prefrontal cortex of subjects with schizophrenia. Am J Psychiatry 2006; 163: 534-7.

[28] Stefansson H, Sigurdsson E, Steinthorsdottir V, et al. Neuregulin 1 and susceptibility to schizophrenia. Am J Hum Genet 2002; 71: 877-92.

[29] Bulayeva KB, Glatt SJ, Bulayev OA, Pavlova TA, Tsuang MT Genome-wide linkage scan of schizophrenia: a cross-isolate study. Genomics 2007; 89: 167-77.

[30] Bulayeva KB, Leal SM, Pavlova TA, et al. Mapping genes of complex psychiatric diseases in Daghestan genetic isolates. Am J Med Genet B Neuropsychiatr Genet 2005; 132: 76-84.

[31] Williams NM, Norton N, Williams $\mathrm{H}$, et al. A systematic genomewide linkage study in 353 sib pairs with schizophrenia. Am J Hum Genet 2003; 73: 1355-67. 